\title{
Sense or sensibility? The neuro-functional basis of the structural matching effect in persuasion
}

\author{
Antonio Aquino $^{1}$ • Francesca Romana Alparone ${ }^{1} \cdot$ Stefano Pagliaro ${ }^{1} \cdot$ Geoffrey Haddock $^{2} \cdot$ Gregory R. Maio $^{3}$. \\ Mauro Gianni Perrucci ${ }^{1,4} \cdot$ Sjoerd J. H. Ebisch ${ }^{1,4}$
}

Published online: 17 March 2020

(C) The Psychonomic Society, Inc. 2020

\begin{abstract}
The present study investigates the neural pathways underlying individual susceptibility to affective or cognitive information in persuasive communication, also known as the structural matching effect. Expanding on the presumed involvement of the ventromedial prefrontal cortex (vMPFC) in persuasion, we hypothesized that the vMPFC contributes to the evaluation of persuasive information depending on its match with the recipient's affective or cognitive predominance. During functional magnetic resonance imaging, 30 participants evaluated 10 consumable products presented with both affective and cognitive persuasive messages. All participants were characterized on a continuum regarding their personal orientation in terms of individual differences in need for affect (NFA) and need for cognition (NFC). The results showed that the vMPFC, posterior cingulate cortex, and cerebellum are more strongly activated when the persuasive message content, either affective or cognitive, matched the recipient's individual affective or cognitive orientation. Interestingly, this effect in the vMPFC was found specifically when participants evaluated the products presented by the persuasive messages, whereas the correlation in the posterior cingulate cortex and cerebellum activity was detected when reading the messages. These results confirm the hypothesis that the vMPFC plays a role in subjectively weighting persuasive message content depending on individual differences in affective and cognitive orientation. Such a structural matching effect might involve the vMPFC particularly during explicit expressions of subjective valuations. These novel findings also further develop the conceptualization of the role of the vMPFC in self-related processing.
\end{abstract}

Keywords Structural matching effect $\cdot$ Self-relevance $\cdot$ Ventral medial prefrontal cortex $\cdot$ Need for affect $\cdot$ Need for cognition

Jane Austen's (1811) classic novel Sense and Sensibility chronicles two sisters: Marianne, who follows her heart, and Elinor, who follows her mind. The story describes a classical dichotomy between individuals who tend to follow their gut feeling and individuals who tend to be more rational. The distinction between the predominance of the heart or the mind in guiding behavior is in accordance with a long line of findings in the psychological literature, indicating that an attitude's basis can be inherently affective - that is, based on

Francesca Romana Alparone

f.alparone@unich.it

1 Department of Neuroscience, Imaging and Clinical Sciences, Chieti-Pescara University, Via dei Vestini 31, 66100 Chieti, Italy

2 Cardiff University, Cardiff, UK

3 University of Bath, Bath, UK

4 Institute of Advanced Biomedical Technologies (ITAB), Chieti-Pescara University, Chieti and Pescara, Italy the positive and/or negative feelings evoked by an objector cognitive - that is, based on beliefs about the positive and/ or negative attributes of an attitude object (e.g., Drolet \& Aaker, 2002; Hovland \& Rosenberg, 1960; Zajonc \& Markus, 1982; Zanna \& Rempel, 1988). Indeed, considerable research has demonstrated that individuals differ in the degree to which their attitudes are based on affective versus cognitive information (for a review, see Haddock \& Maio, 2019).

The distinction between affect and cognition is also apparent in the literature on persuasion. Persuasive appeals can be based on affective (e.g., the emotions elicited by a product) or cognitive properties (e.g., attributes about a product), and research has found that persuasive messages induce greater attitude change when their content matches the affectivecognitive basis of the recipient's attitude (e.g., Fabrigar \& Petty, 1999; Haddock \& Huskinson, 2004; Mayer \& Tormala, 2010). For example, Haddock, Maio, Arnold, and Huskinson (2008) tested whether the impact of affective and cognitive appeals differed as a function of individual differences in the need for affect (NFA; Maio \& Esses, 2001) and 
the need for cognition (NFC; Cacioppo \& Petty, 1982). NFA considers individual differences in the degree to which people approach or avoid situations that induce emotions (Maio \& Esses, 2001). People high in NFA are motivated to understand both their own and others' emotions, and they tend to rely upon emotional information in attitude formation and the regulation of behavior (Haddock \& Huskinson, 2004). In contrast, NFC considers individual differences in the tendency to engage in, and enjoy, complex activities requiring cognitive effort. People high in NFC are more likely to rely upon information about an object's attributes when evaluating it (Cacioppo \& Petty, 1982). Haddock et al. (2008) found that higher levels of NFA predicted greater persuasion in response to an affect-based (but not cognition-based) persuasive message, whereas higher levels of NFC predicted greater persuasion in response to a cognition-based (but not affect-based) persuasive message. They referred to this correspondence as to the structural matching effect, an effect that has been replicated in a number of studies (for a review, see Haddock \& Maio, 2019).

Although there is strong evidence supporting the structural matching effect, evidence for the mechanism(s) underlying this effect is less developed. We believe that such evidence is necessary both at a theoretical level (e.g., regarding the predominance of affect and or cognition in evaluations) and at a practical level (e.g., informing practitioners in the field of persuasion). As part of this development, tracking the neural basis of this matching effect is a novel and important way to further understand its underlying process(es). Toward that end, the current study investigates whether brain activity is differently modulated when people higher in NFA than NFC process persuasive messages that contain either affective or cognitive information.

Our research question intersects with the growing literature on the neural mechanisms underlying persuasion. A number of studies have provided evidence for a crucial role of the medial prefrontal cortex (MPFC) in the processing of persuasive messages (Chua, Liberzon, Welsh, \& Strecher, 2009; Doré, Cooper, Scholz, O'Donnel, \& Falk, 2019; Falk, Berkman, Whalen, \& Lieberman, 2011; Vezich, Katzman, Ames, Falk, \& Lieberman, 2017; see Cacioppo, Cacioppo, \& Petty, 2018, for a review). Importantly, Falk and Scholz (2018) noted that ventral MPFC (vMPFC) activity during persuasion is mainly a consequence of the receiver's subjective evaluation of objects, ideas, and behaviors. In other words, vMPFC activity during persuasion reflects the perceived positive consequences for the message receiver (Fishbein \& Ajzen, 2011; Johnson, Smith-McLallen, Killeya, \& Levin, 2004; O'Keefe, 2013). Falk and Scholz (2018) further highlighted that the receiver's subjective evaluation is mainly predicted by the self-relevance of the message - that is, the integration of the subjective value of the message into the receiver's self-concept. The involvement of the vMPFC in subjective evaluations and in the coding of self-relevance is also confirmed by studies outside the persuasion field (Bartra, McGuire, \& Kable, 2013; Levy \& Glimcher, 2012; Rangel \& Hare, 2010; Sui \& Humphries, 2015). Indeed, a recent metaanalysis (Acikalin, Gorgolewski, \& Poldrack, 2017) found an overlap between a neural subjective value system and the default mode network putatively involved in internal processing and coding of the self-relevance of external information. Consequently, it was suggested that the vMPFC contributes to the subjective valuation process by generating information based on mental simulations.

While research has implicated the vMPFC in the context of persuasion, no studies have investigated (to the best of our knowledge) whether the involvement of the vMPFC in persuasion depends on the correspondence between the affective-cognitive content of persuasive content and individual differences in affective and cognitive orientation. In the present study, participants evaluated 10 everyday consumable products during functional magnetic resonance imaging (fMRI). We reasoned that there should be an increase in perceived self-relevance of the object when information about it matches the recipient's affective or cognitive orientation. Consequently, we expected that individuals with higher NFA than NFC would evaluate the affective messages as more relevant for them compared with the cognitive ones, whereas individuals with higher NFC than NFA would evaluate the cognitive messages as more relevant for them compared with the affective ones.

Further, given the role of the vMPFC in coding selfrelevance and subjective valuation (Acikalin et al., 2017; Northoff \& Hayes, 2011; Qin \& Northoff, 2011), we hypothesized that the vMPFC might support the evaluation of products by affective and cognitive persuasive content depending on individual differences in NFA and NFC. As a result, we predicted stronger vMPFC activity during the evaluation of objects described with affective messages (compared with cognitive ones) in individuals with higher NFA than NFC, with complementary effects for cognitive messages.

Finally, previous research hypothesized a role for the vMPFC in explicit processes rather than in implicit processes, without empirically disentangling its precise role in these selfrelated processes (see, e.g., Moran, Heatherton, \& Kelley, 2009). As applied to the present context, we reasoned that, during the reading phase, people start to implicitly think about the content of the persuasive message, whereas during the evaluation, people reflect on the self-relevance of the stimuli and explicitly express their attitude. As consequence, we explored the role of the vMPFC during both reading and evaluation. We therefore tested whether the vMPFC would be more likely to play a role in the explicit evaluation of the targets than during the reading of the message. If such a differentiation emerges, it would represent a novel and important development in modelling the neuroscience of persuasion (Chua et al., 2009; Falk et al., 2011; Vezich et al., 2017). 


\section{Method}

The data set (along with a guideline for readers), persuasive stimuli, neural maps, and details about the experimental design are available in the Open Science Framework (https://osf. io/xtzad/).

\section{Stimulus development}

To address our research questions, we first had to develop persuasive messages that could be presented in a controlled time frame within an fMRI scanner, while being confident that these messages were affective or cognitive in their basis. We therefore conducted a series of pilot studies.

First, 20 affective and 20 cognitive messages describing consumer products (e.g., a book) were created, using real commercial ads as inspiration. For each product, we generated an affect-based ad and a cognition-based ad. Each message contained five written sentences. The decision to include five written sentences in each persuasive message followed Falk et al.'s (2011) examination of the neuroscience of persuasion. Following past research (e.g., Haddock \& Huskinson, 2004), the affective messages contained terms regarding feelings and sensations induced by the product (e.g., "The soft wool of the pullover 'Tender' gives a fresh scent all day"), whereas the cognitive messages described the product's features and qualities (e.g., "The new full-resistant pullover is made with $100 \%$ merino wool"). Because this was the first study examining the neural pathways recruited in the structural matching effect, all messages were built to elicit positive reactions in order to avoid any possible confound with valence.

The messages were first pretested by asking 64 participants (58 females, six males; $M_{\text {age }}=21.98$ years, $S D=3.09$ years) to evaluate each message on two dimensions. First, participants indicated the degree to which they perceived each message as affective or cognitive $(1=$ very affective, $6=$ very cognitive $)$. Second, they indicated the degree to which they perceived each message as credible $(1=$ not at all credible, $6=$ very credible). Further, as we are unaware of any study that has investigated the relationship of self-relevance with the affective-cognitive content perception of persuasive message, we assessed the role of self-reference in affective-cognitive content perception by asking half of our participants to read messages with self-references (e.g. "The pullover Tender cuddles you in a warm hug"), and the other half to read messages without self-references (e.g. "The pullover Tender cuddles who wears it in a warm hug"). An a priori power analysis was conducted to estimate the desired sample size for this pilot study (using G*Power, Version 3.1; Faul, Erdfelder, Lang, \& Buchner, A., 2007). With an alpha $=.05$ and power $=.95$, the projected minimum sample size needed to detect an effect size of $f=.25$ was 55 for an analysis of variance (ANOVA), with an interaction with a between-participant factor (two levels) and a within-participant factor (two levels).

A 2 (affective versus cognitive) $\times 2$ (self-references versus no self-references) ANOVA showed a significant interaction, $F(1,62)=4.97, p=.029$. An analysis of the simple main effects showed that the difference in the perceived affective and cognitive content of the messages was stronger when they presented self-references $\left(M_{\mathrm{aff}}-M_{\mathrm{cog}}=1.42\right)$, $F(1,62)=86.30, p<.001,95 \%$ CIs for the mean difference $[1.11,1.72], \eta^{2}=.58$, than when they did not $\left(M_{\mathrm{aff}}-M_{\mathrm{cog}}=\right.$ $.95), F(1,62)=44.01, p<.001,95 \%$ CIs for the mean difference $[.66,1.23], \eta^{2}=.41$. These results underlined that self-references are important in the accentuation of affective-cognitive perception differences, and we therefore focused on the messages with self-references. From the messages with self-references, we selected the 10 affective messages and the 10 cognitive messages that differentiated most strongly in terms of affective-cognitive quality perception, based on paired-samples $t$ tests. A $t$ test on the average of the selected affective and cognitive messages confirmed that they differed between the affective-cognitive quality perception, $t(63)=11.94, p<.001, M_{\mathrm{aff}}-M_{\mathrm{cog}}=1.49,95 \% \mathrm{CIs}$ for the mean difference [1.25, 1.73], Cohen's $d=1.49$, but did not differ in credibility, $t(63)=1.44, p=.154, M_{\text {aff }}$ $-M_{\mathrm{cog}}=-.07,95 \%$ CIs for the mean difference $[-.17$, $.03]$, Cohen's $d=.18$. Further, the selected affective and cognitive messages did not differ in total length (mean number of words), $t(9)=.10, p=.918, M_{\mathrm{aff}}-M_{\mathrm{cog}}=.50,95 \%$ CIs for the mean difference $[-10.23,11.23)$, Cohen's $d=$ .18. A set of 20 ( 10 affective and 10 cognitive) messages was chosen to be consistent with other studies on the neuroscience of persuasion (Chua et al., 2009; Falk, Berkman, Mann, Harrison, \& Lieberman, 2010; Vezich et al., 2017). For instance, Chua et al. (2009) used 14 short statements (seven high or low tailored and seven generic cessation statements, or eight high- or low tailored and six generic cessation statements). Falk et al. (2011) used a total of 20 messages to test the neural bases of behavioural change in response to sunscreen use. More recent work by Vezich et al. (2017) used 10 gain messages and 10 loss messages to study the effect of gain versus loss framing in the persuasion.

Next, to further ensure that this subset of messages was perceived as affective or cognitive, they were presented to 22 new participants, who evaluated the extent to which each message was (a) affective or cognitive and (b) credible. Analyses of the ratings reconfirmed the differentiation in perceptions of the affective-cognitive content of the messages, $t(21)=6.09, p<.001, M_{\mathrm{aff}}-M_{\mathrm{cog}}=1.64,95 \%$ CIs for the mean difference [1.11, 2.17], Cohen's $d=1.30$. Results also indicated that the affective $\left(M_{\text {aff }}=4.06, S D=.56\right)$ and cognitive $\left(M_{\operatorname{cog}}=4.25, S D=.48\right)$ messages were rated as equally credible, $t(21)=1.54, p=.137, M_{\mathrm{aff}}-M_{\mathrm{cog}}=-.19,95 \% \mathrm{CIs}$ for the mean difference $[-.43, .05]$, Cohen's $d=.30$. 
Finally, to control for the duration of the presentation of each persuasive message, each message was vocally registered at a normal pace. Subsequently, we presented 10 new participants with all of the messages to ascertain that the timing was sufficient to read and understand the messages. From this pretest, it emerged that the established duration did not differ between the affective $(M=36,180 \mathrm{~ms}, S D=$ $6,086 \mathrm{~ms})$ and cognitive ( $M=37,800 \mathrm{~ms}, S D=4,800 \mathrm{~ms})$ messages, $t(19)=-1.17, p=.271,95 \%$ CIs for the mean difference $[-4,741.78,1,501.78]$, Cohen's $d=.26$.

\section{Main experiment}

\section{Participants}

Sample size was determined using fMRIPower (SPM Toolkit; Mumford \& Nichols, 2008), specialized software used to determine power estimates for functional MRI (fMRI) data based on maps from studies with similar experimental designs. We selected data from an fMRI study by van der Laan, de Ridder, Viergever, and Smeets (2014), which examined the neural patterns associated with food choices using a contrast between two experimental conditions and covariation with an individual differences measure, with an effect size for whole brain analyses of .80 . Based on these representative data, fMRIPower revealed that a sample of 32 participants would be required for a statistical power of .80 .

Thirty-four participants took part in the present fMRI experiment. Four participants were excluded from fMRI analysis because their head motion exceeded the movement parameter limit of the size of one voxel $(3 \mathrm{~mm})$ in both runs of the experimental design (Power, Barnes, Snyder, Schlaggar, \& Petersen, 2013). No participants exceeded this motion limit in only one fMRI run.

Consequently, the retained sample included 30 participants ( 15 females, 15 males) between the age of 20 and 32 years ( $M$ $=25.10$ years, $S D=3.08$ years). All participants had normal or corrected-to-normal vision and reported no history of neurological or psychiatric disease or reading difficulties. All participants provided written informed consent in compliance with the Helsinki Declaration and guidelines of the local Ethical Committee (Protocol No. 898).

\section{Materials and procedure}

\section{Pre-fMRI}

Prior to fMRI scanning, we assessed participants' levels of NFA and NFC. Participants' NFA was assessed with the short version of the NFA scale (Appel, Gnambs, \& Maio, 2012). This scale comprises 10 items: five items measure the motivation to approach emotions (e.g., "Emotions help people to get along in life," $\alpha=.83$ ), and five assess the motivation to avoid emotions (e.g., "I do not know how to handle my emotions, so I avoid them," $\alpha=.81$ ). Participants responded to these statements on a 7 -point scale $(1=$ totally disagree; $7=$ totally agree). A single NFA score was calculated by summing responses after reverse scoring the avoidance items $(M=5.55$, $S D=.69$, range of observed scores: 4.10-6.50).

Participants' NFC was assessed using the 18-item NFC scale (Cacioppo, Petty, \& Kao, 1984). Participants rated the extent to which they agreed with items such as "I really enjoy a task that involves coming up with new solutions to problems" and "Thinking is not my idea of fun" (reverse scored). Participants responded to these statements on a 7-point scale $(1=$ extremely uncharacteristic of me; $7=$ extremely characteristic of me). A single NFC score was calculated by summing responses after reverse scoring the negatively keyed items $(M=4.96, S D=.63$, range of observed scores: $3.50-6.00$ ).

For both conceptual and methodological reasons, we operationalized the personal orientation of the participants as the difference between standardized NFA and NFC scores (range: $-1.89-1.79$ ). From a conceptual perspective, we were interested in examining the relative reliance on affect versus cognition (see also Aquino, Haddock, Maio, Wolf, \& Alparone, 2016). From a methodological perspective, conceptualizing individual differences in the form of a difference score leads to statistical models with more degrees of freedom in their error terms. Consequently, a higher NFA-NFC score indicated a higher reliance on affect, whereas a lower score indicated a higher reliance on cognition. The distribution of the standardized NFA-NFC scores across participants is available in Table 1 of the Supplementary Materials.

\section{fMRI procedure}

After the assessment of NFA and NFC, all participants underwent an fMRI scan session. During fMRI scanning, we monitored participants' neural activity while they were visually presented with the affective (e.g., "Choosing the Backpack 'Poke' makes you feel all the potentialities of life in a joyful party of colors and makes you feel the excitement of a new journey where every direction is possible. 'Poke' marks the rhythm of the most exciting experiences of your life and does it with overwhelming energy. 'Poke' also offers endless possibilities to express your personality and to be surprised by unique and innovative solutions. Over the years, it has become a symbol of discovery, euphoria, and freedom for all generations. 'Poke' is a real icon of contemporary style, with an exciting story to tell") and cognitive persuasive messages (e.g., "The 'Caps' backpack is very handy and comfortable thanks to the many internal pockets that allow you to carry everything you need. Its dimensions allow you to carry it as hand luggage on all main airlines. The 'Caps' backpack is also equipped with a very useful inner lining that protects your notebook from hits and rain. Ergonomic shoulder bag and 
filled seatback make it one of the most comfortable backpacks on the market. 'Caps' shows an original front closure with leather strips and it is also equipped with a hidden magnet closure"). Participants were informed that during the scan session they would read 20 messages (the pretested 10 affective and 10 cognitive messages) and that subsequently, they would evaluate each of the objects presented in the messages (see Fig. 1).

The affective and the cognitive messages were presented in a randomized order in two fMRI runs. During the reading phase, participants were asked to read each message attentively. The duration for the reading phase was set on the basis of the pretest that was conducted to ascertain that the time for the reading was sufficient for participants and to ascertain that the affective and cognitive messages did not differ in duration. This reading time was also adapted to be a multiple of the TR (i.e., 1,800 ms; the average reading time across the messages was $34,960 \mathrm{~ms}$ ).

The reading phase was always followed by an explicit evaluation phase: After a randomly varying interval $(1,800-$
5,400 ms), participants expressed their attitude by rating how much they liked the object, on a scale ranging from 1 (not at all) to 7 (very much). This evaluation was reported after each individual message. In addition, after another randomly varying interval (1,800-5,400 ms), we assessed intentions to buy the described object by asking participants how likely it was that they would buy the object in the next 3 weeks on a scale ranging from 1 (not at all) to 7 (very likely). This intention rating was reported after each individual message. All participants had a time limit of 5,400 ms to express their attitudes and intentions.

Participants expressed their answers by pressing buttons that allowed them to increase (right button press with the right middle finger; maximum $=7$ ) or decrease (left button press with right index finger; minimum $=1$ ) the starting value of 4 that appeared on the screen. We registered the value expressed at the end of the time limit. Given the high correlation between attitudes and intentions, $r(30)=.96, p<.001$, we averaged these judgments to create a single index labelled evaluation.

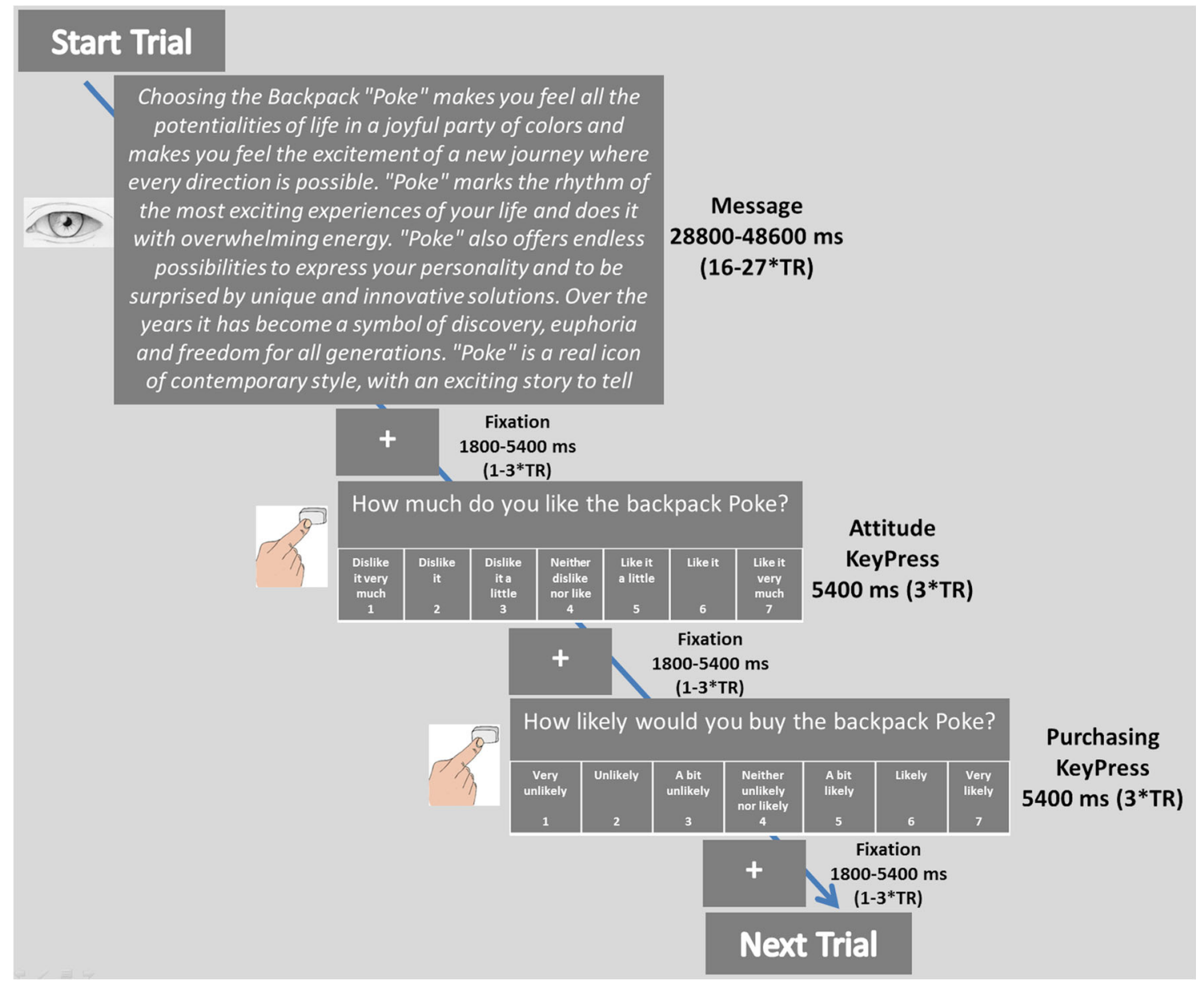

Fig. 1 Schematic of the task with the relative timeline and fMRI time window. The affective and the cognitive messages were presented in a randomized order in two fMRI runs (each run included five affective and five cognitive messages). During the reading phase (message in the figure), participants were asked to read each message attentively. After a randomly varying interval $(1,800-5,400 \mathrm{~ms}$, fixation in the figure), participants expressed their attitude and their purchasing intention (spaced out by a fixation cross). Note. TR $=$ repetition time used for fMRI $(1,800 \mathrm{~ms})$ 


\section{fMRI data acquisition}

Imaging data were acquired using a 3 Tesla MR scanner (Philips Achieva X Series; Philips Medical System, Best, The Netherlands) at the Institute of Advanced Biomedical Technologies (ITAB) in Chieti, Italy. A sensitivity-encoding eight-channel brain coil was used. Head motion was minimized using foam padding and surgical tape; the pushbutton was fixed in place using surgical tape connected to the scanner bed. An initial T1-weighted anatomical (3-D TFE pulse sequence) was acquired with the following parameters: field of view $=240$ $\mathrm{mm}$; voxel size $=(1 \mathrm{~mm} \times 1 \mathrm{~mm} \times 1 \mathrm{~mm}) ; \mathrm{TR}=8.1 \mathrm{~ms}$; TE $=3.7 \mathrm{~ms}$. Subsequently, two task fMRI runs (number of fMRI frames: 404 for the first run and 397 for the second run) were acquired in a single fMRI session (T2* weighted EPI sequence with $\mathrm{TR}=1,800 \mathrm{~ms} ; \mathrm{TE}=30 \mathrm{~ms} ; 35$ slices; slice thickness $=$ $3.5 \mathrm{~mm}$; in-plane voxel size $=3 \mathrm{~mm} \times 3 \mathrm{~mm}$; field of view $=$ $228 \times 122 \times 240 \mathrm{~mm}$; flip angle $=85$ ).

\section{fMRI preprocessing and analysis}

Raw fMRI data were analyzed with Brain Voyager QX 2.3 software (Brain Innovation, Maastricht, The Netherlands). Preprocessing of functional data included slice scan time correction, motion correction, and removal of linear trends from voxel time series. Three-dimensional motion correction was performed with a rigid body transformation to match each functional volume to the reference volume, estimating three translations and three rotation parameters. We excluded fMRI runs with head motion exceeding the size of one voxel (i.e., $>3 \mathrm{~mm}$ ) from analysis (Power et al., 2013). Preprocessed functional volumes of each participant were coregistered with the corresponding structural data set. Because the 2-D functional and 3-D structural measurements were acquired in the same session, the coregistration transformation was determined using the slice position parameters of the functional images and the position parameters of the structural volume. Structural and functional volumes were transformed into the Talairach space (Talairach $\&$ Tournoux, 1988) using a piecewise affine and continuous transformation. Functional volumes were resampled at a voxel size of $3 \mathrm{~mm} \times 3 \mathrm{~mm} \times 3 \mathrm{~mm}$ and spatially smoothed with a Gaussian kernel of $6 \mathrm{~mm}$ full-width half maximum to account for intersubject variability.

As a preliminary analysis, we explored whether the affective-cognitive messages could be associated with differential neural activity depending on the persuasive content (i.e., feeling-associated sentences or cognition-associated sentences). To examine this question, we performed a voxelwise whole-brain analysis. A paired-samples $t$ test (affective versus cognitive) according to a random effect model was applied to directly compare the affective and cognitive message reading as well as evaluation. Monte Carlo simulations (1,000 simulations) were performed to access the overall significance level of group statistical maps (thresholded at $p$ $<.001)$ and to determine a cluster size threshold $(k)$ in order to obtain group statistical maps with a significance threshold of $p=.01$ after cluster-level correction for multiple comparisons (Cox, 1996; Forman et al., 1995). This analysis was run separately for the reading and for the evaluation phases.

To test whether differential neural activity between the processing of affective and cognitive messages was correlated with individual differences in affective-cognitive orientation (i.e., NFA minus NFC), region of interest analyses were performed. For this purpose, a priori independent spherical ROIs with a radius of $7 \mathrm{~mm}$ were created based on the coordinates from a recent relevant meta-analysis showing an overlap between the default mode network and the subjective value network in vMPFC (Acikalin et al., 2017; Talairach coordinates after transformation from Montreal Neurological Institute [MNI] space according to Lancaster et al., 2007: vMPFC BA10/32: $-0.2,38.4,4.8$ ).

Moreover, as a control procedure, we also considered the additional regions described in the meta-analysis of Acikalin et al. (2017) associated with overlapping activity across the subjective value and the default network, although they might cover slightly different functions in attitudinal processes. For instance, according to Acikalin et al. (2017), the information elaborated in the vMPFC might be subsequently integrated in the dorsal posterior cingulate cortex (dPCC), constituting another main component of both the subjective value system and the default mode network (Talairach coordinates after transformation from MNI space according to Lancaster et al., 2007: PCC BA31: -2.7, -39.9, 33.1; PCC BA23: -3.6, -52, 14.8). Other regions included the striatum (Talairach coordinates after transformation from MNI space according to Lancaster et al., 2007: Striatum 1: 0.5, 9.7, -1.8; Striatum 2: -8.4 , $21.5,1.8$; Striatum $3:-5.6,19.6,1.7$ ), and the bilateral amygdala (right amygdala: $22.4,-5.5,-12.3$; left amygdala: -19.8 , $-5.2,-12.7)$. Thus, a total number of eight ROIs was considered for the analysis.

For each participant, neural activity during the exposure to the affective and cognitive trials (i.e., subtraction of the average beta values for the affective and cognitive condition of the same product, e.g., pullover, book, etc., across all the voxels included in each ROI) was extracted from the ROIs, while the difference score on the NFA and the NFC scales was used as a covariate (NFA minus NFC). Correlation analyses were performed including 1,000 bootstrap samples to obtain $95 \%$ confidence intervals (CIs), and results were corrected for multiple comparisons using Bonferroni correction (eight ROIs). The analyses were run separately for the two experimental phases: reading and evaluation. As control analyses, we assessed whether the results were stable across different radii; thus, we repeated the same analyses with a radius of $5 \mathrm{~mm}$ and with a radius of $9 \mathrm{~mm}$. Given that no differences emerged across different radii, for parsimonious reasons, we only present the 
ROI-based results during the reading and the evaluation phase by focusing on the ROIs with an average radius of $7 \mathrm{~mm}$.

In order to explore whether regions different from the expected ROIs correlated with affective-cognitive orientation, the relationship between the differential neural activity from the processing of affective versus cognitive messages and the individual differences in affective-cognitive orientation (NFA minus NFC) was also investigated using a voxel-wise wholebrain analysis. This analysis was also performed separately for the reading and explicit evaluation phases to test the effect of the covariate (individual orientation) on brain activity. The statistical significance of this whole-brain covariance analysis was assessed by means of paired-samples $t$ tests according to a random-effects model. Monte Carlo simulations (1,000 simulations) were performed to assess the overall significance level of group statistical maps (thresholded at $r=.50$ ) and to determine a cluster size threshold $(k)$ in order to obtain group statistical maps with a significance threshold of $p=.01$ after cluster level correction for multiple comparisons (Cox, 1996; Forman et al., 1995).

\section{Post-fMRI behavioral measures}

At the end of the fMRI scanning section, we asked participants to re-read the persuasive messages presented during the previous fMRI scan. For each pair of messages (i.e., affective and cognitive) about an object, participants were asked to indicate which of the two messages was more relevant for them and which of the two messages they preferred. Participants expressed their response by using a 6-point Likert scale from cognitive message to affective message $(1=$ absolutely the affective message, $6=$ absolutely the cognitive message; the affective-cognitive anchors were balanced across the $10 \mathrm{ob}-$ jects). The two questions were repeated for all 10 objects used in our experimental fMRI study. The order of presentation of the stimuli was randomized across the 30 participants. A total score was computed so that a higher score indicated that participants perceived the affective messages as more relevant for them and that they preferred the affective messages more than the cognitive ones.

\section{Results}

\section{Preliminary analyses}

The preliminary whole-brain voxel-wise paired-samples $t$ test between the reading of affective and cognitive messages revealed a lateralized activation pattern in the brain (see Fig. 2a). Reading the affective messages, compared with the cognitive ones, more strongly activated the right hemisphere insula (Brodmann area [BA] 13), fusiform gyrus (BA 37), superior temporal (BA 22), and extrastriate cortex (BA 19).
Conversely, reading the cognitive messages, compared with the affective ones, more strongly activated the left hemisphere insula (BA 13), superior temporal (BA 22), middle temporal (BA 21), hippocampus (BA 54), fusiform gyrus (BA 37), and extrastriate cortex (BA 19); $t(29)>3.66, p<.01$ corrected, $k>$ 209, Cohen's $d=.67$.

A similar pattern emerged by contrasting the explicit evaluation of affective and cognitive messages, $t(29)>3.66, p<$ .01 (corrected), $k>43$ (see Fig. 2b). The conjunction analysis identifying brain regions modulated by affective-cognitive message content during both reading and explicit evaluation phases showed overlapping increased activity for the processing of affective messages in the right hemisphere BA 13, BA 22 , BA 19, and for the processing of the cognitive messages in the left hemisphere, BA 21, BA 13, BA 19 and BA 37; $t(29)>$ $3.66, p<.01$ (corrected), $k>59$ (see Fig. 2c), Cohen's $d=.67$.

\section{The structural matching effect test: Reading phase}

Starting from the reading phase, the ROI-based analyses showed that no ROIs correlated with the difference in the affective-cognitive orientation, not even the vMPFC (see Fig. 3). The ROI-based results for the reading phase are presented in Table 1 (upper section of the table). The scatterplots of the correlations between the affective-cognitive orientation and neural activity in the ROIs and during both the reading and the evaluation phase are provided in the Supplementary Materials.

While the ROI-based analysis did not yield significant correlations for the PCC ROIs, the voxel-wise whole-brain analysis showed during the reading phase a statistically significant relationship between affective versus cognitive orientation and neural activity in the ventral posterior cingulate cortex (vPCC; BA 23) and in the cerebellum $(r>.50, p<0.01$, corrected, $k>16)$. No significant correlation was detected in vMPFC in the voxel-wise whole-brain analysis. Specifically, this covariance analysis revealed that individuals with higher NFA (than NFC) showed stronger activation of both the vPCC and the cerebellum when they read persuasive affective messages (compared with the cognitive ones). The whole-brain voxel-wise results are presented in Table 2 and Fig. 4.

\section{The structural matching effect test: Explicit evaluation phase}

The ROI-based covariance analysis in the explicit evaluation phase showed that neural activity in the vMPFC increased with higher participant' NFA (than NFC) when they explicitly evaluated the affective messages (compared with the cognitive ones; see Fig. 3). Similarly, the correlational analysis highlighted that neural activity in this ROI increased among participants with higher NFC (than NFA) when they explicit evaluated the cognitive messages, $r=.501, p=.040$, 


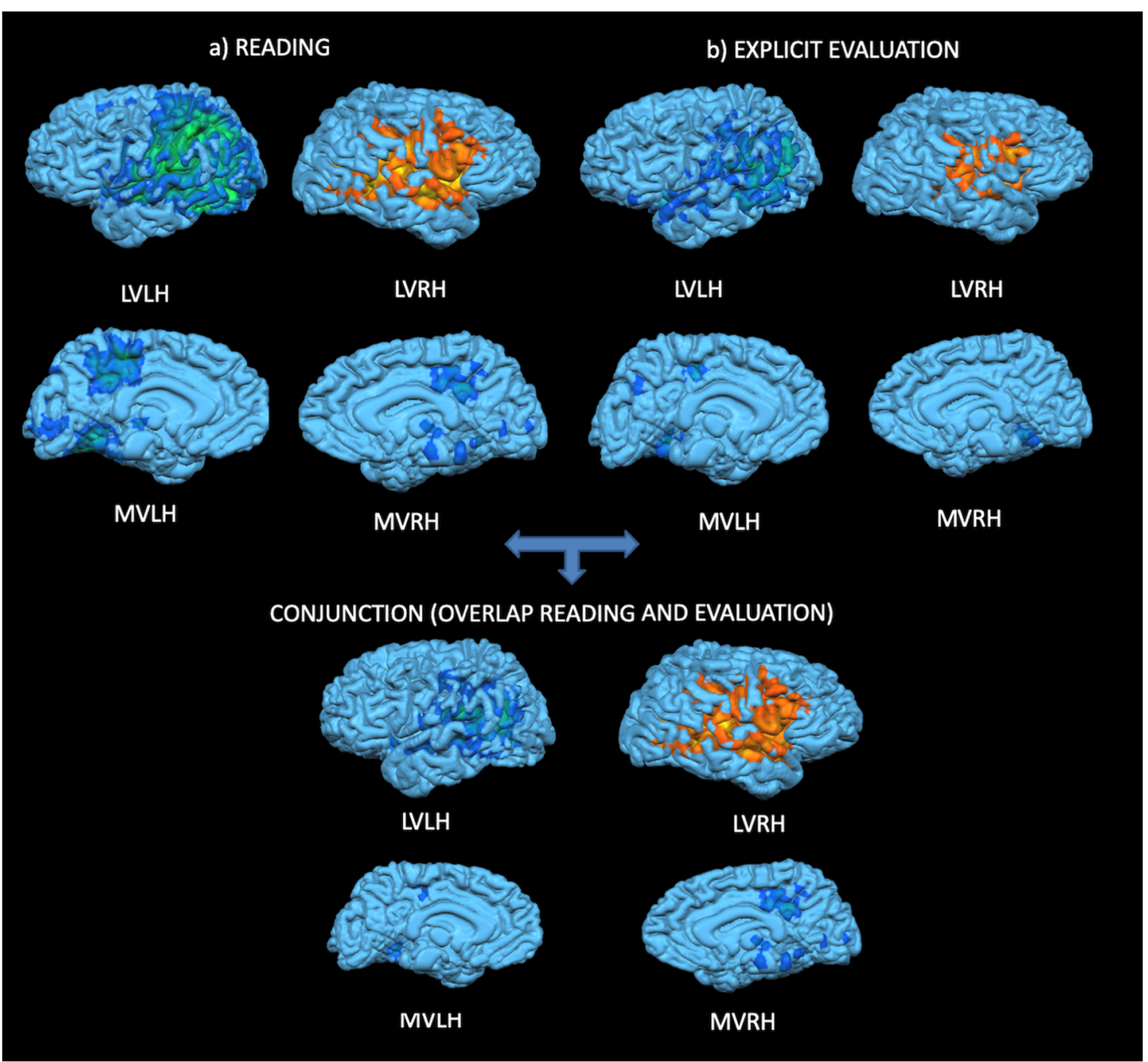

Fig. 2 Group statistical maps of whole brain voxel-wise $t$ test between affective versus cognitive message conditions during (a) reading, (b) explicit evaluation, and (c) spatially overlapping for the affective versus cognitive message conditions during the two phases (reading + explicit evaluation). The figure shows the lateral view of the left hemisphere

Bonferroni corrected; $S E=.15 ; 95 \%$ CIs $=[.15, .74]$. The ROI-based results are presented in Table 1 (lower section of the Table). The activation of the ROI followed the normal distribution without statistical outliers in the sample (index of kurtosis: .07; index of skewness: .57, Shapiro-Wilk test for normality: .96, $p=.28$ )

The vMPFC was the only ROI that correlated with the difference in affective-cognitive orientation. Taken together, these findings confirmed that the VMPFC has a crucial role in the explicit evaluation, which is consistent with the fact that this is the outcome of the matching effect.

The whole-brain voxel-wise paired-samples $t$ test comparing the evaluation of affective and cognitive messages with the NFA-NFC difference score as a covariate (NFA minus NFC score) revealed that no regions different from the
(LVLH), the lateral view of the right hemisphere (LVRH), the medial view of the left hemisphere (MVLH), and the medial view of the right hemisphere (MRVH). Red/yellow indicates stronger activity for the affective message conditions, whereas blue/green indicates stronger activity for the cognitive message conditions. (Color figure online)

vMPFC were activated in affective and cognitive people in response to the matched message $(r>.50, p<.005$, uncorrected, $k>10$; see Table 2).

Examining the activation of the vMPFC during the evaluation in more detail (see Table 2), it can be noted that the mean response to the affective and cognitive stimuli is negative or slightly above zero (see Discussion for an explanation of this finding).

\section{Post-fMRI behavioral measurements}

As hypothesized, the difference in personal orientation (NFA minus NFC) was positively related to measures of self-relevance, $r(29)=.53, p=.003, S E=.12,95 \%$ CI $[.26, .73]$. In other words, people with an affective orientation considered 
a)

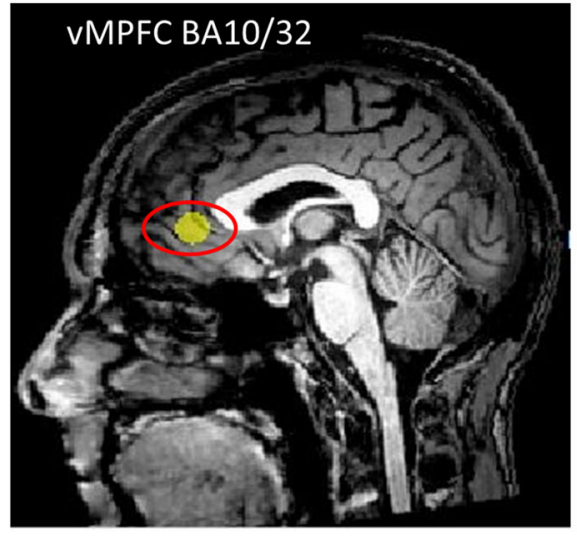

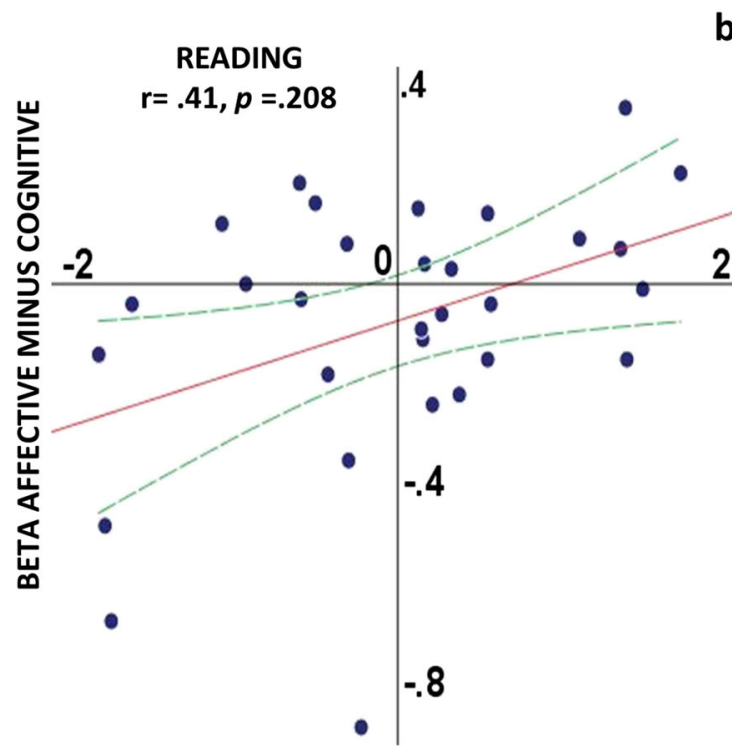

NFA MINUS NFC

Fig. 3 a A priori ROI (sphere) based on meta-analyses results from Acikalin et al. (2017), radius: $7 \mathrm{~mm}$ ). b Scatter plots visualizing the relationship between differential beta values in the ROI for affective and cognitive message processing (beta affective minus cognitive), and differential b)

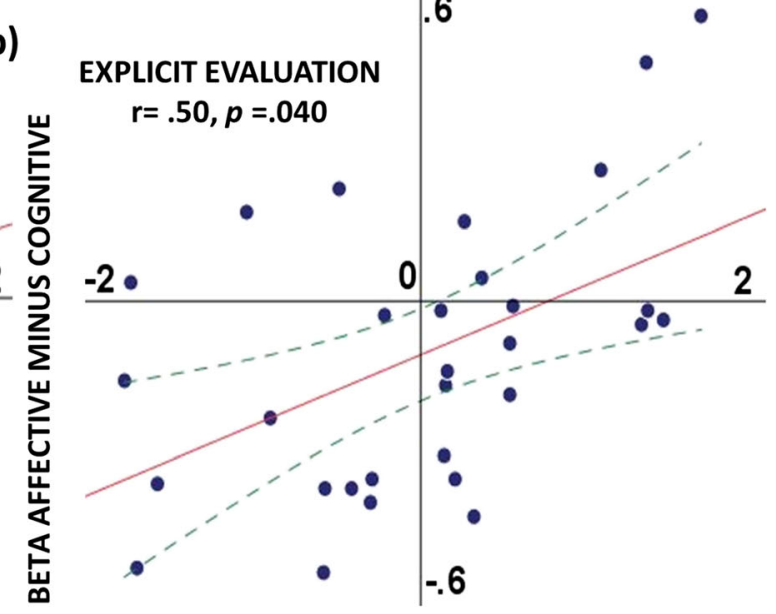

NFA MINUS NFC

affective-cognitive orientation (need for affect [NFA] minus need for $\operatorname{cog}$ nition $[\mathrm{NFC}]$ ), and correlation coefficients ( $p$ values Bonferroni corrected for multiple comparisons) during both reading and evaluation phase. Red line $=$ line of best fit; shaded green lines $=95 \%$ CIs. (Color figure online)

Table 1 Correlations of neural activity in the ROIs with affective-cognitive personal orientation (need for affect [NFA] minus need for cognition [NFC]) during the (a) reading and (b) explicit evaluation

\begin{tabular}{|c|c|c|c|c|c|c|c|c|}
\hline & VMPFC & Striatum1 & Striatum2 & Striatum3 & PCC1 & PCC2 & Amygdala Right & Amygdala Left \\
\hline \multicolumn{9}{|l|}{$\mathrm{a}$} \\
\hline \multicolumn{9}{|l|}{ READING } \\
\hline \multirow[t]{4}{*}{ NFA minus NFC } & $r=.41$ & $r=.21$ & $r=.26$ & $r=.31$ & $r=-.16$ & $r=-.10$ & $r=.11$ & $r=-.08$ \\
\hline & $p=.208$ & $p=1$ & $p=1$ & $p=.832$ & $p=1$ & $p=1$ & $p=1$ & $p=1$ \\
\hline & $S E=.15$ & $S E=.16$ & $S E=.16$ & $S E=.16$ & $S E=.23$ & $S E=.20$ & $S E=.20$ & $S E=.18$ \\
\hline & CI $[-.07, .69]$ & CI $[-.17, .49]$ & $\mathrm{CI}[-.05, .56]$ & $\mathrm{CI}[-.01, .61]$ & CI $[-.60, .26]$ & CI $[-.49, .30]$ & CI $[-.33, .39]$ & $\mathrm{CI}[-.27, .36]$ \\
\hline \multicolumn{9}{|c|}{ 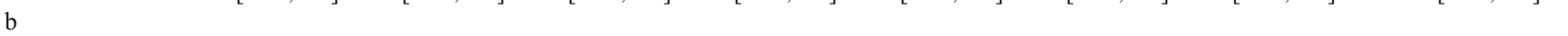 } \\
\hline \multicolumn{9}{|l|}{ EVALUATION } \\
\hline \multirow[t]{4}{*}{ NFA minus NFC } & $r=.50$ & $r=.27$ & $r=.18$ & $r=.30$ & $r=-.01$ & $r=-.02$ & $r=.28$ & $r=-.06$ \\
\hline & $p=.040$ & $p=1$ & $p=1$ & $p=.88$ & $p=1$ & $p=1$ & $p=1$ & $p=1$ \\
\hline & $S E=.15$ & $S E=.13$ & $S E=.18$ & $S E=.17$ & $S E=.17$ & $S E=.16$ & $S E=.19$ & $S E=.17$ \\
\hline & CI $[.15, .74]$ & CI $[-.01, .54]$ & $\mathrm{CI}[-.19, .55]$ & CI $[-.07, .60]$ & $\mathrm{CI}[-.34, .35]$ & $\mathrm{CI}[-.32, .31]$ & CI $[-.12, .60]$ & CI $[-.27, .36]$ \\
\hline
\end{tabular}

Note. The associated significance levels are Bonferroni corrected for multiple comparisons. $\mathrm{CI}=$ confidence interval (95\% based on bootstrapping with 1,000 simulations) 
Table 2 Brain regions showing a significant relationship between participants' affective versus cognitive orientation and their neural activity during the fMRI task, yielded by the whole-brain voxel-wise analyses

\begin{tabular}{llllll}
\hline Brain region & $\begin{array}{l}\text { Peak coordinates } \\
(x, y, z)\end{array}$ & Peak $r$ & $\begin{array}{l}\text { Mean affective } \\
(S E)\end{array}$ & Mean cognitive $(S E)$ & Cluster size \\
\hline PCC & $12,-62,19$ & .66 & $-.19(.03)$ & $-.20(.03)$ \\
(reading) & & & $.19(.06)$ & $.30(.07)$ & $.08(.13)$ \\
Cerebellum (reading) & $-13,-64,-19$ & .64 & $-.05(.12)$ & $-.07(.02)$ & 532 \\
vMPFC 1 & $13,34,-2$ & .57 & $-1.04(.17)$ & & \\
(evaluation) & $-2,44.2$ & .54 & & & \\
vMPFC2 & & & & & \\
(evaluation) & & & & & \\
\hline
\end{tabular}

Note. The mean and standard error $(S E)$ represents the BOLD mean responses as a function of the affective and the cognitive quality of the messages
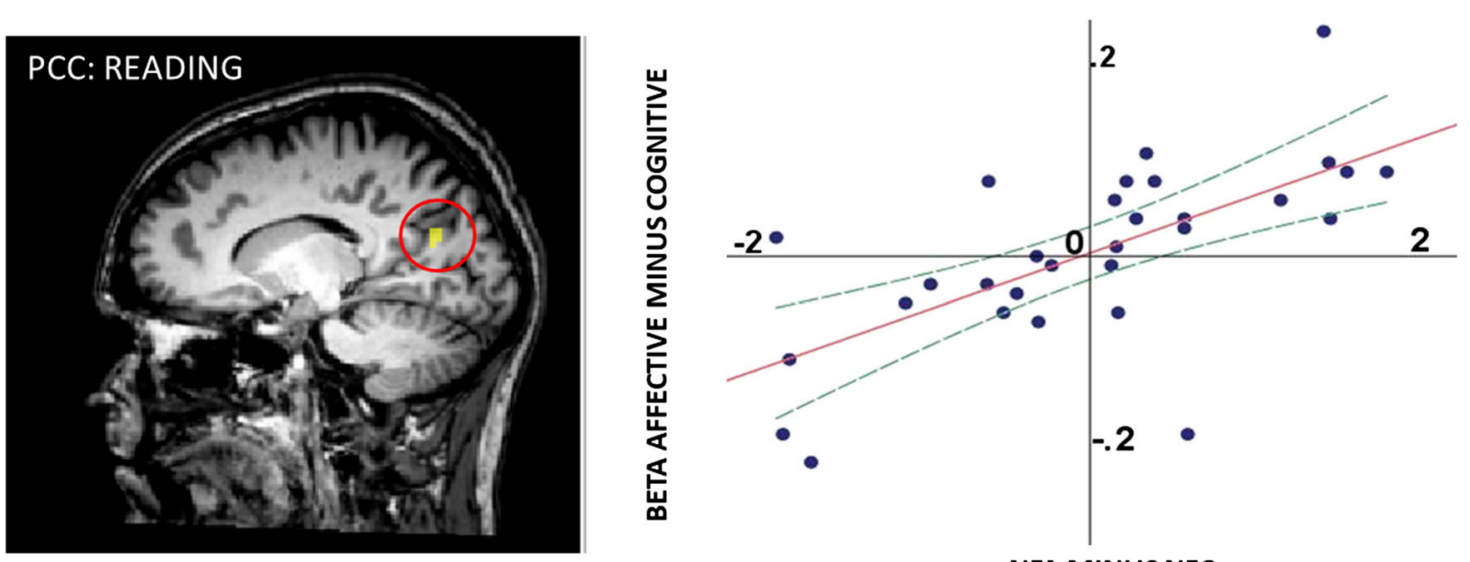

NFA MINUS NFC

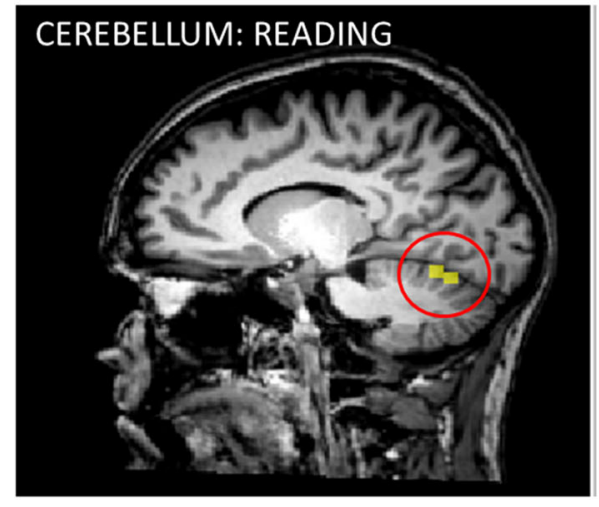

a)

Fig. 4 a Voxel clusters $(r>.50, p<.01$, corrected) showing significant covariance between differential beta values for affective and cognitive message processing (beta affective minus cognitive), and differential affective-cognitive orientation during reading. $\mathbf{b}$ Scatter plots visualizing the relationship between differential beta values in the clusters for

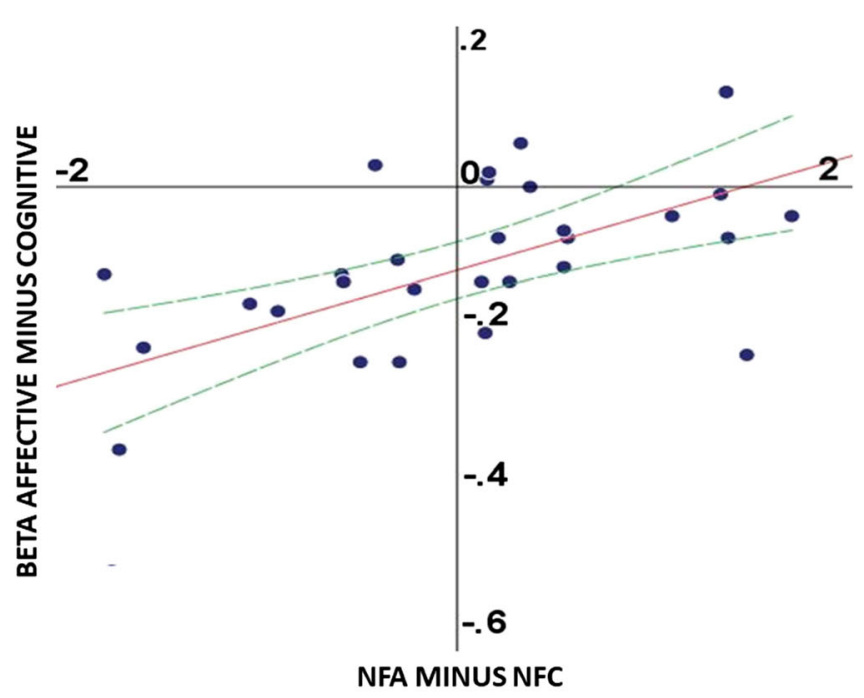

b)

affective and cognitive condition (beta affective minus cognitive), and differential affective-cognitive orientation (need for affect [NFA] minus need for cognition $[\mathrm{NFC}]$ ). Red line $=$ line of best fit; shaded green lines $=$ 95\% CIs. (Color figure online) 
the affective messages (compared with the cognitive ones) as more personally relevant for them and they preferred the affective messages compared with the cognitive ones. The selfrelevance measure was not related to the evaluation, $r(29)=$ $.27, p=.15, S E=.19,95 \% \mathrm{CI}[-.13, .59]$, indicating that these two measures are tapping different constructs.

\section{Discussion}

\section{The neural basis of the matching effect}

There is a growing literature on the neural mechanisms underlying persuasion, but significant questions remain about the neural processes underlying the affective-cognitive structural matching effect. To begin to understand these processes, we used fMRI to examine neural activity during the evaluation of objects presented with affective versus cognitive persuasive messages. Our findings demonstrated that the vMPFC is more strongly activated when people evaluate a product presented by a persuasive message that matched the recipient's affective versus cognitive orientation. That is, we found stronger activity in the vMPFC for affective messages (compared with the cognitive ones) among individuals with an affective focus, whereas activity in the VMPFC was stronger for cognitive messages (compared with the affective ones) among individuals with a cognitive focus. These findings extend prior observations that the vMPFC is involved in persuasion (Chua et al., 2009; Falk et al., 2011; Falk \& Scholz, 2018) by showing that this region is also important to the structural matching effect.

Not only do these results provide the first evidence of neural differences in responding to affective versus cognitive persuasion as a function of individual differences, but the centering of these differences in the vMPFC helps to articulate the mechanism underlying these effects. Given the putative role of the vMPFC in coding the self-relevance of external stimuli (Levy \& Glimcher, 2012; Northoff \& Hayes, 2011; Rangel \& Hare, 2010), a possible interpretation of our results is that persuasive messages become self-relevant for perceivers when message content matches individuals' preference for affect or cognition, identifying the state of the judge in relation to the object of judgement (Zajonc, 1980). This interpretation is supported by our post-fMRI behavioral measures showing that individuals with an affective orientation perceived the affective messages as more personally relevant for them, compared with the cognitive ones, and that the affective people preferred the affective messages compared with the cognitive ones. Relevant to this assumption, accurate stimulus preparation and testing prior to the fMRI experiment helped to ensure that self-references were balanced across affective and cognitive messages in our study.

Interestingly, we found that vMPFC activity correlated with affective versus cognitive orientation during the explicit evaluation of corresponding targets, whereas no significant correlation was detected during the preceding reading of affective and cognitive information. These findings imply that the role of the VMPFC in persuasion is mainly associated with the explicit evaluation process rather than mere reading of persuasive content. This provides an important and innovative contribution to the debate about spontaneous and deliberative processing of self-relevant information (Moran et al., 2009).

Of additional relevance, the vMPFC is located within a socalled task-negative network showing strong default activity during a resting state (i.e., the absence of specific tasks or stimuli) and decreases of this activity during most tasks (Di Plinio \& Ebisch, 2018; Raichle et al., 2001). For this reason, this network has been associated with free thought and self-related processing (Andrews-Hanna, Smallwood, \& Spreng, 2014), mental activities in which participants could be especially involved when not performing structured cognitive tasks. From this point of view, the structural matching effect in the vMPFC suggests less deviation from this "default" state of activity when message content matches personal orientation.

Notably, our data indicated that the structural matching effect was linked with the vMPFC, but not the dPCC or other structures of the subjective value system. As suggested by Acikalin et al. (2017), these structures, in particular, the vMPFC and $\mathrm{APCC}$, although constituting a subjective value system, might cover somewhat different functions in the process that goes from attitude change to behaviour change as a consequence of a persuasive message. However, the wholebrain analysis showed enhanced activation of the vPCC and cerebellum during the reading of affective messages (compared with the cognitive ones) in individuals higher in NFA than NFC. The covariance of PCC activity with individual orientation during the reading of the persuasive message involved a more ventral and posterior area compared with the ROI described by Acikalin et al. (2017). Previous literature has shown that the $\mathrm{vPCC}$ is activated during tasks that require an internal focus of attention, such as autobiographical memory retrieval (Svoboda, McKinnon, \& Levine, 2006) and envisioning the future (Schacter et al., 2012). In the current context, we can speculate that during the reading phase, participants relate the content of the messages to their personal past experience; the vPCC could serve such a temporal bridge, while the vMPFC supports a transient valuation in terms of self-relevance. Nevertheless, our data do not allow us to support this claim directly, and future studies need to further clarify this intriguing relationship between the $\mathrm{VPCC}$ and the vMPFC during different phases of persuasion.

Regarding the involvement of the cerebellum in the reading phase, this might be due to its role in attention processes. In particular, the cerebellum allows attention to be shifted rapidly and accurately (Allen, Buxton, Wong, \& Courchesne, 1997). As applied to the current context, indirect support for this hypothesis is provided by Haddock et al. (2008), who found 
that people with a stronger affective orientation processed affective messages more deeply than cognitive messages (and vice versa for people with a stronger cognitive orientation). Furthermore, a number of efferent and afferent connections of the cerebellum with several other brain structures have been described, such as the dorsolateral and dorsomedial prefrontal cortices, portions of the posterior parietal cortex, the superior temporal region, the thalamus, and the limbic system (Krienen \& Buckner, 2009; Middleton \& Strick, 2000; Riva \& Giorgi, 2000; Schmahmann \& Sherman, 1998). Given its localization and connections, the cerebellum likely contributes also to decision-making processes (Cardoso et al., 2014; Ernst et al., 2002; Lawrence, Jollant, O’ Daly, Zelaya, \& Phillips, 2009). In this regard, Blackwood, Ffytche, Simmons, Bentall, Murray, and Howard, (2004) reported activation of the cerebellum during decision making under circumstances of both certainty and uncertainty, but it was more pronounced in conditions of uncertainty. The authors suggested that the cerebellum plays a role in the internal representation of uncertain events, facilitating the prediction of future outcomes as well as inductive processes. We can speculate that during the reading phase, participants generated an internal representation of the content message by joining the content with their personal experiences followed by its evaluation in terms of subjective value (i.e., activation of the vMPFC in the evaluation phase). Future studies are needed to elucidate the connection between vPCC, cerebellum, and vMPFC during the evaluation.

\section{The affective-cognitive lateralization in persuasion}

An ancillary result of the present research regards the specialization of the right hemisphere for the processing of affective messages and a specialization of the left hemisphere for the processing of cognitive messages. A wide literature has already highlighted the predominance of the right hemisphere in the elaboration of emotional words (Killgore \& Yurgelun-Todd, 2007; Schwartz, Davidson, \& Maer, 1975), as well as the predominance of the left hemisphere for sentence comprehension (Geschwind, 1972; Sakai, Tatsuno, Suzuki, Kimura, \& Ichida, 2005), which can be considered a cognitive process. Our research further supports the lateralization hypothesis, making a direct comparison between affective and cognitive persuasive information. To our knowledge, these data demonstrate that hemisphere specialization occurs during both the reading of affective versus cognitive messages and during the evaluation of objects previously described by these messages.

\section{Limitations and future directions}

We wish to acknowledge the potential limitations of our results. First, spherical ROIs were based on the coordinates reported by an independent previous meta-analysis of functional neuroimaging data examining brain regions associated with specific, relevant functions. It could be argued that the use of spherical ROIs may not represent the most adequate tool for testing hypotheses in functional neuroimaging. In fact, Eickhoff, Heim, Zilles, and Amunts (2006) showed that an anatomical ROI approach (i.e., maximum probability maps), has major advantages over the traditional spheres approach used to define ROIs. That is, a definition based on probabilistic anatomical maps is independent from the analyzed functional data and thus represents a genuine a priori hypothesis. Further, in contrast to the spherical ROIs, which require decisions by the investigator, the anatomical ROIs approach is completely objective. This may improve the comparability of results from different studies. Another advantage of the anatomical ROIs approach is that this method avoids arbitrary decisions about radii and related multiple comparison corrections. In the present research, we assessed whether the results were stable across different ROI radii (i.e., $5 \mathrm{~mm}, 7 \mathrm{~mm}, 9$ $\mathrm{mm}$ ) to ensure that the findings were not specific for a determined ROI radius. However, this strategy increases the number of comparisons and consequently brings about issues with proper Type I error control and increases the chance to obtain false positive results. Although it remains methodologically challenging to accurately integrate functional neuroimaging data, like those reported by meta-analyses on specific functions, with anatomical ROIs, future studies should test whether the neural bases of the matching effect in persuasion are replicated using an anatomical ROI approach.

Secondly, a potential alternative explanation for our brain specialization findings is the valence hypothesis (Adolphs, Jansari, \& Tranel, 2001). This hypothesis proposes that the left cerebral hemisphere is specialized for processing positive emotions and the right hemisphere is specialized for processing negative emotions. However, this hypothesis cannot be tested by our data, because our participants were exposed to stimuli designed to elicit only positive attitudes. Future studies might focus on the valence of the appeals by including positive and negative message content. Similarly, we are not able to provide any direct information about the link between the structural matching effect, as reflected by the functional properties of the vMPFC, and the attitudinal self-report measures. Nevertheless, the results provide intriguing input for further studies to investigate the behavioral relevance of vMPFC activity. An important issue to consider is whether having participants complete the NFA and NFC before the scan (rather than after) primed their responses to be more self-consistent throughout the fMRI task. However, this possibility is inconsistent with previous studies revealing no effect of the order of presentation of personal orientation on the matching effect (see Haddock \& Maio, 2019, for a review).

Finally, the sample size is a relevant issue in neuroimaging (Geuter, Qi, Welsh, Wager, \& Lindquist, 2018). In this paper (Geuter et al., 2018), the authors showed that sample sizes of 40 are generally able to detect regions with high effect 
sizes (Cohen's $d>0.8$ ), while sample sizes closer to 80 are required to reliably recover regions with medium effect sizes $(0.5<d<0.8)$. However, the sample size $(N=30)$ of the present fMRI study can be considered above the average size for task-based fMRI studies on similar topics (Chua et al., 2009; Falk et al. 2011, Vezich et al., 2017), and other control analyses (confidence intervals based on bootstrapping) confirmed that the detected correlations between vMPFC activity and individual orientation were robust.

\section{Conclusions}

Our results show that vMPFC activity depends on the match between persuasive message content and individual differences in attitude bases. These novel findings significantly contribute to research on both attitude formation and persuasion, as well as social neuroscience more broadly. From the social neuroscience perspective, our findings help to show that people rely differentially upon affective and cognitive information in persuasive messages, depending on their preferences for affective and cognitive information. This role of individual differences provides an important counterpoint for thinking about the persuasion process in the mainstream literature, which even today sustains the prevalence of affectiveemotional appeals over cognitive ones in attitude change (e.g., Rocklage, Rucker, \& Nordgren, 2018). Our results show that people vary in their weighting of affective and cognitive information, and these differences are identifiable in relevant neural activity. From a cognitive perspective, our results further reinforce the idea that the self-relevance is not an intrinsic characteristic of the message, but can change depending on the receivers of the persuasive communication and their affective-cognitive orientation in particular (see Doré et al., 2019).

Returning to Sense and Sensibility (Austen, 1811), we believe that our findings resonate with how Marianne and Elinor's differences in reliance on heart versus mind remains a powerful theme in the present day because such individual differences are basic and prevalent in how people respond to social information, and indeed are traceable to distinct patterns of neural activity in response to affective and cognitive cues. Through the possession of distinguishable routes to processing affective versus cognitive information in the brain, we are capable of differing in our reliance on these sources of information with interesting and important consequences (e.g., Marianne and Elinor's choice of suitor).

Acknowledgements We thank Daniele Paolini, Valeria Amata Giannella, and the neuroradiology staff at the ITAB for their assistance during the data collection. This work was partially supported by the Departments of Excellence 2018-2022 initiative of the Italian Ministry of Education, University and Research for the Department of Neuroscience, Imaging and Clinical Sciences (DNISC) of the University of Chieti-Pescara.
Open practices statements The data set (along with a guideline for readers), persuasive stimuli, neural maps, and details about the experimental design are available in the Open Science Framework (https://osf.io/xtzad/).

Author contributions Francesca Romana Alparone and Antonio Aquino conceived the research idea and developed the research design in collaboration with Sjoerd J. H. Ebisch. Antonio Aquino, and Francesca Romana Alparone collected and analyzed along with Sjoerd J. H Ebisch, Stefano Pagliaro, and Mauro Gianni Perrucci. All the authors contributed to interpreting the data and writing the paper.

\section{References}

Acikalin, M. Y., Gorgolewski, K. J., \& Poldrack, R. A. (2017). A coordinate-based meta-analysis of overlaps in regional specialization and functional connectivity across subjective value and default mode networks. Frontiers in Neuroscience, 11, 1-11. https://doi.org/ 10.3389/fnins.2017.00001

Adolphs, R., Jansari, A., \& Tranel, D. (2001). Hemispheric perception of emotional valence from facial expressions. Neuropsychology, 15, 516-524. https://doi.org/10.1037/0894-4105.15.4.516

Allen, G., Buxton, R. B., Wong, E. C., \& Courchesne, E. (1997). Attentional activation of the cerebellum independent of motor involvement. Science, 275, 1940-1943. https://doi.org/10.1126/ science.275.5308.1940

Andrews-Hanna, J. R., Smallwood, J., \& Spreng, R. N. (2014). The default network and self-generated thought: Component processes, dynamic control, and clinical relevance. Annals of the New York Academy of Sciences, 1316, 29-52. https://doi.org/10.1111/nyas. 12360

Appel, M., Gnambs, T., \& Maio, G. R. (2012). A short measure of the need for affect. Journal of Personality Assessment, 94, 418-426. https://doi.org/10.1080/00223891.2012.666921

Aquino, A., Haddock, G., Maio, G. R., Wolf, L. J., \& Alparone, F. R. (2016). The role of affective and cognitive individual differences in social perception. Personality and Social Psychology Bulletin, 42, 798-810. https://doi.org/10.1177/0146167216643936

Austen, J. (1811). Sense and sensibility. New York, NY: Oxford University Press.

Bartra, O., McGuire, J. T., \& Kable, J. W. (2013). The valuation system: A coordinate-based meta-analysis of BOLD fMRI experiments examining neural correlates of subjective value. NeuroImage, 76, 412427. https://doi.org/10.1016/j.neuroimage.2013.02.063

Blackwood, N., Ffytche, D., Simmons, A., Bentall, R., Murray, R., \& Howard, R. (2004). The cerebellum and decision making under uncertainty. Cognitive Brain Research, 20, 46-53. https://doi.org/ 10.1016/j.cogbrainres.2003.12.009

Cacioppo, J. T., Cacioppo, S., \& Petty, R. E. (2018). The neuroscience of persuasion: A review with an emphasis on issues and opportunities. Social Neuroscience, 13, 129-172. https://doi.org/10.1080/ 17470919.2016.1273851

Cacioppo, J. T., \& Petty, R. E. (1982). The need for cognition. Journal of Personality and Social Psychology, 42,116-131. https://doi.org/10. 1037/0022-3514.42.1.116

Cacioppo, J. T., Petty, R. E., \& Feng Kao, C. (1984). The efficient assessment of need for cognition. Journal of Personality Assessment, 48, 306-307. https://doi.org/10.1207/s15327752jpa4803_13

Cardoso, C. D. O., Branco, L. D., Cotrena, C., Kristensen, C. H., Schneider Bakos, D. D. G., \& Fonseca, R. P. (2014). The impact of frontal and cerebellar lesions on decision making: Evidence from the Iowa gambling task. Frontiers in Neuroscience, 8, 61. https:// doi.org/10.3389/fnins.2014.00061 
Chua, H. F., Liberzon, I., Welsh, R. C., \& Strecher, V. J. (2009). Neural correlates of message tailoring and self-relatedness in smoking cessation programming. Biological Psychiatry, 65, 165-168. https:// doi.org/10.1016/j.biopsych.2008.08.030

Cox, R. W. (1996). AFNI: Software for analysis and visualization of functional magnetic resonance neuroimages. Computers and Biomedical Research, 29, 162-173. https://doi.org/10.1006/cbmr.1996.0014

Di Plinio, S., \& Ebisch, S. J. (2018). Brain network profiling defines functionally specialized cortical networks. Human Brain Mapping, 39, 4689-4706. https://doi.org/10.1002/hbm.24315

Doré, B. P., Cooper, N., Scholz, C., O’Donnell, M. B., \& Falk, E. B. (2019). Cognitive regulation of ventromedial prefrontal activity evokes lasting change in the perceived self-relevance of persuasive messaging. Human Brain Mapping, 40, 1-10. https://doi.org/10. 1002/hbm. 24545

Drolet, A., \& Aaker, J. (2002). Off-target? Changing cognitive-based attitudes. Journal of Consumer Psychology, 12, 59-68. https://doi. org/10.1207/S15327663JCP1201 0

Eickhoff, S. B., Heim, S., Zilles, K., \& Amunts, K. (2006). Testing anatomically specified hypotheses in functional imaging using cytoarchitectonic maps. NeuroImage, 32 (2), 570-582.

Ernst, M., Bolla, K., Mouratidis, M., Contoreggi, C., Matochik, J. A., Kurian, V., ... London, E. D. (2002). Decision-making in a risktaking task: A PET study. Neuropsychopharmacology, 26, 682691. https://doi.org/10.1016/s0893-133x(01)00414-6

Fabrigar, L. R., \& Petty, R. E. (1999). The role of the affective and cognitive bases of attitudes in susceptibility to affectively and cognitively based persuasion. Personality and Social Psychology Bulletin, 25, 363-381. https://doi.org/10.1177/ 0146167299025003008

Falk, E., \& Scholz, C. (2018). Persuasion, influence, and value: Perspectives from communication and social neuroscience. Annual Review of Psychology, 69, 329-356. https://doi.org/10.1146/ annurev-psych-122216-011821

Falk, E. B., Berkman, E. T., Mann, T., Harrison, B., \& Lieberman, M. D. (2010). Predicting persuasion-induced behavior change from the brain. Journal of Neuroscience, 30(25), 8421-8424.

Falk, E. B., Berkman, E. T., Whalen, D., \& Lieberman, M. D. (2011). Neural activity during health messaging predicts reductions in smoking above and beyond self-report. Health Psychology, 30, 177-185. https://doi.org/10.1037/a0022259

Faul, F., Erdfelder, E., Lang, A. G., \& Buchner, A. (2007). G* Power 3: A flexible statistical power analysis program for the social, behavioral, and biomedical sciences. Behavior Research Methods, 39, 175-191. https://doi.org/10.3758/bf03193146

Fishbein, M., \& Ajzen, I. (2011). Predicting and changing behavior: The reasoned action approach. New York, NY: Psychology Press.

Forman, S. D., Cohen, J. D., Fitzgerald, M., Eddy, W. F., Mintun, M. A., \& Noll, D. C. (1995). Improved assessment of significant activation in functional magnetic resonance imaging (fMRI): Use of a clustersize threshold. Magnetic Resonance in Medicine, 33, 636-647. https://doi.org/10.1002/mrm.1910330508

Geschwind, N. (1972) Language and brain. Scientific American 226, 76 83. https://doi.org/10.1038/scientificamerican0472-76

Geuter, S., Qi, G., Welsh, R. C., Wager, T. D., \& Lindquist, M. A. (2018). Effect size and power in fMRI group analysis. Biorxiv, 295048. https://doi.org/10.1101/295048

Haddock, G., \& Huskinson, T. L. H. (2004). Individual differences in attitude structure. In G. Haddock \& G. R. Maio (Eds.), Contemporary perspectives on the psychology of attitudes (pp. 35-56). London, England: Psychology Press. https://doi.org/10. 4324/9780203645031

Haddock, G., \& Maio, G. R. (2019). Inter-individual differences in attitude content: Cognition, affect, and attitudes. Advances in Experimental Social Psychology, 59, 53-102. https://doi.org/10. 1016/bs.aesp.2018.10.002
Haddock, G., Maio, G. R., Arnold, K., \& Huskinson, T. (2008). Should persuasion be affective or cognitive? The moderating effects of need for affect and need for cognition. Personality and Social Psychology Bulletin, 34, 769-778. https://doi.org/10.1177/0146167208314871

Hovland, C. I., \& Rosenberg, M. J. (1960). Attitude organization and change. New Haven, CT: Yale University Press.

Johnson, B. T., Smith-McLallen, A., Killeya L. A., \& Levin, K. D. 2004. Truth or consequences: Overcoming resistance to persuasion with positive thinking. In. E. S. Knowles \& J. A. Linn (Eds), Resistance and persuasion (pp. 215-33). New York, NY: Psychology Press. https://doi.org/10.4324/9781410609816

Killgore, W. D., \& Yurgelun-Todd, D. A. (2007). The right-hemisphere and valence hypotheses: Could they both be right (and sometimes left)? Social Cognitive and Affective Neuroscience, 2, 240-250. https://doi.org/10.1093/scan/nsm020

Krienen, F. M., \& Buckner, R. L. (2009) Segregated fronto-cerebellar circuits revealed by functional connectivity. Cerebral Cortex, 19, 2485-2489. https://doi.org/10.1093/cercor/bhp135

Lancaster, J. L., Tordesillas-Gutiérrez, D., Martinez, M., Salinas, F., Evans, A., Zilles, K., ..., Fox, P. T. (2007). Bias between MNI and Talairach coordinates analyzed using the ICBM-152 brain template. Human Brain Mapping, 28, 1194-1205. https://doi.org/10.1002/ hbm. 20345

Lawrence, N. S., Jollant, F., O’Daly, O., Zelaya, F., \& Phillips, M. L. (2009). Distinct roles of prefrontal cortical subregions in the Iowa gambling task. Cerebral Cortex, 19, 1134-1143. https://doi.org/10. 1093/cercor/bhn154

Levy, D. J., \& Glimcher, P. W. (2012). The root of all value: a neural common currency for choice. Current Opinion in Neurobiology, 22, 1027-1038. https://doi.org/10.1016/j.conb.2012.06.001

Maio, G. R., \& Esses, V. M. (2001). The need for affect: Individual differences in the motivation to approach or avoid emotions. Journal of Personality, 69, 583-614. https://doi.org/10.1111/14676494.694156

Mayer, N. D., \& Tormala, Z. L. (2010). "Think" versus "feel” framing effects in persuasion. Personality and Social Psychology Bulletin, 36, 443-454. https://doi.org/10.1177/0146167210362981

Middleton, F. A., \& Strick, P. L. (2000). Basal ganglia and cerebellar loops: Motor and cognitive circuits. Brain Research Reviews, 31, 236-250. https://doi.org/10.1016/s0165-0173(99)00040-5

Moran, J. M., Heatherton, T. F., \& Kelley, W. M. (2009). Modulation of cortical midline structures by implicit and explicit self-relevance evaluation. Social Neuroscience, 4, 197-211. https://doi.org/10. 1080/17470910802250519

Mumford, J. A., \& Nichols, T. E. (2008). Power calculation for group fMRI studies accounting for arbitrary design and temporal autocorrelation. NeuroImage, 39, 261-268. https://doi.org/10.1016/j. neuroimage.2007.07.061

Northoff, G., \& Hayes, D. J. (2011). Is our self nothing but reward? Biological Psychiatry, 69, 1019-1025. https://doi.org/10.1016/j. biopsych.2010.12.014

O'Keefe, D. J. (2013). The relative persuasiveness of different forms of arguments-from-consequences: A review and integration. Annals of the International Communication Association, 36, 109-135. https:// doi.org/10.1080/23808985.2013.11679128

Power, J. D., Barnes, K. A., Snyder, A. Z., Schlaggar, B. L., \& Petersen, S. E. (2013). Steps toward optimizing motion artifact removal in functional connectivity MRI: A reply to Carp. NeuroImage, 76, 439-441. https://doi.org/10.1016/j.neuroimage.2012.03.017

Qin, P., \& Northoff, G. (2011). How is our self related to midline regions and the default-mode network?. NeuroImage, 57, 1221-1233. https://doi.org/10.1016/j.neuroimage.2011.05.028

Raichle, M. E., MacLeod, A. M., Snyder, A. Z., Powers, W. J., Gusnard, D. A., \& Shulman, G. L. (2001). A default mode of brain function. Proceedings of the National Academy of Sciences of the United States of America, 98, 676-682. https://doi.org/10.1073/pnas.98.2.676 
Rangel, A., \& Hare, T. (2010). Neural computations associated with goaldirected choice. Current Opinion in Neurobiology, 20, 262-270. https://doi.org/10.1016/j.conb.2010.03.001

Riva, D., \& Giorgi, C. (2000). The cerebellum contributes to higher functions during development: Evidence from a series of children surgically treated for posterior fossa tumors. Brain, 123, 1051-1061. https://doi.org/10.1093/brain/123.5.1051

Rocklage, M. D., Rucker, D. D., \& Nordgren, L. F. (2018). Persuasion, emotion, and language: The intent to persuade transforms language via emotionality. Psychological Science, 29, 749-760. https://doi. org/10.1177/0956797617744797

Sakai, K. L., Tatsuno, Y., Suzuki, K., Kimura, H., \& Ichida, Y. (2005). Sign and speech: Amodal commonality in left hemisphere dominance for comprehension of sentences. Brain, 128, 1407-1417. https://doi.org/10.1093/brain/awh465

Schacter, D. L., Addis, D. R., Hassabis, D., Martin, V. C., Spreng, R. N., \& Szpunar, K. K. (2012). The future of memory: Remembering, imagining, and the brain. Neuron, 76, 677-694. https://doi.org/10. 1016/j.neuron.2012.11.001

Schmahmann, J. D., \& Sherman, J. C. (1998). The cerebellar cognitive affective syndrome. Brain, 121, 561-579. https://doi.org/10.1093/ brain/121.4.561

Schwartz, G. E., Davidson, R. J., \& Maer, F. (1975). Right hemisphere lateralization for emotion in the human brain: Interactions with cognition. Science, 190, 286-288. https://doi.org/10.1126/science.1179210

Sui, J., \& Humphreys, G. W. (2015). The integrative self: How selfreference integrates perception and memory. Trends in Cognitive Sciences, 19, 719-728. https://doi.org/10.1016/j.tics.2015.08.015
Svoboda, E., McKinnon, M. C., \& Levine, B. (2006). The functional neuroanatomy of autobiographical memory: A meta-analysis. Neuropsychologia, 44, 2189-2208.

Talairach, J., \& Tournoux, P. (1988). Co-planar stereotaxic atlas of the human brain: 3-dimensional proportional system: an approach to cerebral imaging. Stuttgart, NY: Thieme.

van der Laan, L. N., de Ridder, D. T., Viergever, M. A., \& Smeets, P. A. (2014). Activation in inhibitory brain regions during food choice correlates with temptation strength and self-regulatory success in weight-concerned women. Frontiers in Neuroscience, 8. https:// doi.org/10.3389/fnins.2014.00308

Vezich, I. S., Katzman, P. L., Ames, D. L., Falk, E. B., \& Lieberman, M. D. (2017). Modulating the neural bases of persuasion: Why/how, gain/loss, and users/non-users. Social Cognitive and Affective Neuroscience, 12, 283-297. https://doi.org/10.1093/scan/nsw113

Zajonc, R. B. (1980). Feeling and thinking: Preferences need no inferences. American Psychologist, 35, 151-175. https://doi.org/10. 1037//0003-066x.35.2.151

Zajonc, R. B., \& Markus, H. (1982). Affective and cognitive factors in preferences. Journal of Consumer Research, 9, 123-131. https://doi. org/10.1086/208905

Zanna, M. P., \& Rempel, J. K. (1988). Attitudes: A new look at an old concept. In D. Bar-Tal, \& A. W. Kruglanski (Eds.), The social psychology of knowledge (pp. 315-334). New York, NY: Cambridge University Press.

Publisher's note Springer Nature remains neutral with regard to jurisdictional claims in published maps and institutional affiliations. 